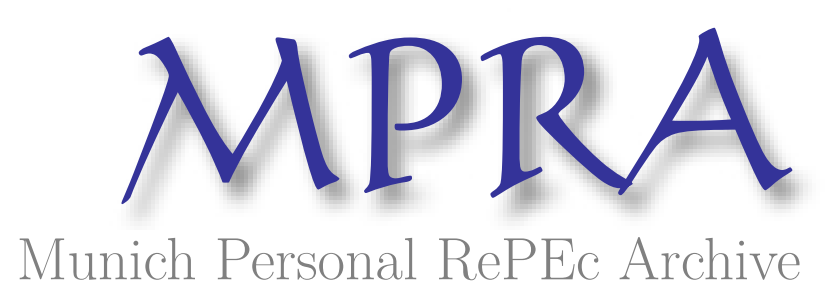

\title{
A Note on the Dynamics of Incentive Contracts
}

Sun, Ching-jen

Deakin University

August 2007

Online at https://mpra.ub.uni-muenchen.de/9813/

MPRA Paper No. 9813, posted 05 Aug 2008 00:37 UTC 


\title{
A Note on the Dynamics of Incentive Contracts
}

\author{
Ching-jen Sun* \\ Department of Economics \\ The Ohio State University
}

February 24, 2008

*Department of Economics, The Ohio State University, 464 Arps Hall, 1945 North High Street, Columbus, OH 43210. Tel.: (614)292-5584, e-mail: sun.161@osu.edu. I am grateful to James Peck for his guidance. I would also like to acknowledge Howard P. Marvel, Huanxing Yang and Lixin Ye for their valuable comments. All remaining errors are my own. 


\begin{abstract}
Laffont and Tirole [3] show that when the uncertainty about the agent's ability is small, the equilibrium must involve a large amount of pooling, but it is not necessary to be a partition equilibrium. They construct a nonpartition continuation equilibrium for a given first-period menu of contracts and conjecture that this continuation equilibrium need not be suboptimal for the whole game under small uncertainty. We show that, irrespective of the amount of uncertainty, this nonpartition continuation equilibrium generates a smaller payoff for the principal than a different menu of contracts with a partition continuation equilibrium. In this sense, Laffont and Tirole's menu of contracts, giving rise to a nonpartition continuation equilibrium, is not optimal. An intuition behind this result is provided that may shed some light on the problem of dynamic contracting without commitment.

Keywords: Incentive Contracts; Dynamic Contracting; Commitment; Partition Equilibrium; Ratchet Effect; Bunching.
\end{abstract}

JEL classification: D86; L51 


\section{Introduction}

In a pioneering paper that extends their static analysis of cost regulation (Laffont and Tirole [2]) to a dynamic framework, Laffont and Tirole [3] study a two-period principal/agent model in which the principal cannot commit himself to second-period actions and the agent's type space is a continuum. In the first period, the principal offers an incentive scheme and observes the agent's performance (cost), which depends on the agent's ability and first-period effort (both of which are unobservable). In the second period, the principal is allowed to update the incentive scheme, and the agent is free to accept the new scheme or to quit. Even though they are not able to characterize the whole set of feasible incentive schemes and solve for the optimal contract, some important properties are derived. First, they show that for any given first-period incentive scheme, there exists no separating continuation equilibrium; hence, some pooling is necessary in equilibrium. Moreover, they show that when the uncertainty about the agent's ability is small, the equilibrium must involve a large amount of pooling (to be defined in the next section), but it is not necessary to be a partition equilibrium. They construct a nonpartition continuation equilibrium for a given first-period menu with two contracts and conjecture that this continuation equilibrium need not be suboptimal for small uncertainty.

We demonstrate that, whether the uncertainty is small or not, Laffont and Tirole's menu of contracts, giving rise to a nonpartition continuation equilibrium, is not optimal. To do this, we construct two first-period incentive schemes leading to partition continu- 
ation equilibria and show that one of the two partition continuation equilibria gives the principal higher payoffs. The intuition of our proof comes from the trade-off between efficiency distortion and the information rent effect.

Nearly two decades after their publication, no one has been able to fully characterize the whole set of incentive contracts and solve for the optimal dynamic contract. The difficulty arises from the failure of the revelation principle in repeated relationships without commitment caused by the ratchet effect. Recognizing the difficulty of probing for a full characterization of equilibria, some authors focus their analyses on dynamic contracting without commitment in a restricted class of equilibria. Caillaud and Mezzetti [1] study equilibrium reserve prices in sequential ascending auctions. They analyze the equilibrium reserve prices in the set of "equilibria with separation under participation." An equilibrium with separation under participation is a perfect Bayesian equilibrium in which in the first period bidders with valuations above some threshold $v^{*}$ follow a symmetric, strictly increasing bidding strategy, and bidders with valuations below $v^{*}$ do not participate. ${ }^{1}$ They show that in equilibrium the seller chooses the first-auction reserve price to induce a positive measure of bidder types to participate, but the measure of participating types is smaller than in a one-shot auction. Sun [5] studies dynamic price discrimination and quality provision with customer recognition. Within the class of partition equilibria, he shows that in equilibrium the firm finds it optimal to offer a single contract in the first period when the social surplus function is log submodular or

\footnotetext{
${ }^{1}$ Notice that the partition property of the continuation equilibrium is implied by the assumption of symmetry, as all types who choose not to participate are below $v^{*}$.
} 
consumers are patient. Moreover, if the firm's optimal strategy is to offer a single contract in the first period, then the firm chooses to serve fewer consumers than in the static setup, and some consumers downgrade the product in the second period. Hence solving for the optimal contract in repeated relationships without commitment is possible if we impose the symmetry or partition assumption in equilibrium. However, can we justify restricting the analysis to the set of partition equilibria? Some possible justifications will be discussed in the next section.

This note proceeds as follows. In the next section, we introduce the static model and summarize the main results obtained by Laffont and Tirole [2]. In section 3, we briefly introduce the dynamic model and present the nonpartition equilibrium constructed by Laffont and Tirole, and show that it is suboptimal. Section 4 concludes.

\section{The Static Model}

Consider a regulator who wants to ask a firm to implement a valuable indivisible project. The cost of the project depends on the firm's efficiency type $\beta$ and the effort level $e$ performed by the firm's manager. Specifically, the cost structure of the project is

$$
c=\beta-e
$$

The regulator can observe the realized $\operatorname{cost} c$, but not the firm's type $\beta$ or effort level $e$. The firm knows its own type $\beta$, and the regulator believes that $\beta$ is distributed on $[\underline{\beta}, \bar{\beta}]$ with strictly positive density $f(\beta)$ and cumulative distribution $F(\beta)$, all of

which is common knowledge. We assume that the hazard rate is monotone, i.e., $\frac{F(\beta)}{f(\beta)}$ is 
nondecreasing. Define a contract as a transfer-cost pair $(s, c)$ that specifies the monetary transfer $s$ the firm can get by carrying out the project at cost $c$. With a slight abuse of notation, we use $s(c)$ to represent the transfer in the pair $(s, c)$. Let $\mathcal{C} \subset \mathbb{R}^{2}$ denote the menu of contracts offered by the regulator. For a given menu $\mathcal{C}$, a firm with type $\beta$ chooses a contract in $\mathcal{C}$ (and, hence, the effort level $e$ as they are one-to-one) to maximize the transfer, $s(c)$, less the disutility of effort, $\psi(e)=\psi(\beta-c)$, where $\psi^{\prime}>0$ and $\psi^{\prime \prime}>0$. For a given $\mathcal{C}$, define the profit function for type $\beta$ as $\Pi(\beta)=\max _{(s, c) \in \mathcal{C}} s(c)-$ $\psi(\beta-c),(s(c(\beta)), c(\beta))$ a corresponding contract that maximizes firm $\beta^{\prime} s$ profit, i.e., $(s(c(\beta)), c(\beta)) \in \arg \max _{(s, c) \in \mathcal{C}} s(c)-\psi(\beta-c)$, and $e(\beta)=\beta-c(\beta)$. A firm with type $\beta$ takes a contract iff the individual rationality constraint $\Pi(\beta) \geq 0$ is met; otherwise the firm exercises its exit option to quit.

Let $u$ be the social utility of the project. The gross payment made by the regulator to the firm is $s+c$. Assume that for each unit of money raised by the regulator, there is a distortionary cost $\lambda>0$ (it could be the cost from levying taxes). The regulator's problem is to choose $\mathcal{C} \subset \mathbb{R}^{2}$ to maximize the social surplus: ${ }^{2}$

$$
\begin{aligned}
W & =\max _{C \subset R^{2}} \int_{\underline{\beta}}^{\bar{\beta}}[u-(1+\lambda)[s(c(\beta))+c(\beta)]+\Pi(\beta)] d F(\beta) \\
\text { s.t. } \Pi(\beta) & =s(c(\beta))-\psi(e(\beta)) \geq 0, \beta \in[\underline{\beta}, \bar{\beta}]
\end{aligned}
$$

By invoking the revelation principle, the envelope theorem (Milgrom and Segal [4]) and integration by parts, we obtain the following results that will be used frequently in the proof of Lemma 1:

\footnotetext{
${ }^{2}$ Assume that the project is always worth carrying out from the point of view of the regulator.
} 
Proposition 1 (Laffont and Tirole [2]) (i) The regulator's problem is equivalent to picking a nondecreasing function $e(\cdot):[\underline{\beta}, \bar{\beta}] \rightarrow \mathbb{R}$ to maximize the following objective function:

$$
W^{*}=\max W=\int_{\underline{\beta}}^{\bar{\beta}}\left\{[u-(1+\lambda)(\psi(e(\beta))+\beta-e(\beta))] f(\beta)-\lambda \psi^{\prime}(e(\beta)) F(\beta)\right\} d \beta
$$

(ii) The optimal solution $e^{*}(\beta)$ solves the following equation

$$
\psi^{\prime}\left(e^{*}(\beta)\right)=1-\frac{\lambda}{1+\lambda} \frac{F(\beta)}{f(\beta)} \psi^{\prime \prime}\left(e^{*}(\beta)\right)
$$

\section{Example of a Nonpartition Equilibrium}

Now we briefly introduce the two-period version of the static model and discuss Laffont and Tirole's example of a nonpartition equilibrium. The timing of the two-period game is as follows: (i) In the first period, the regulator chooses a menu of contracts $\mathcal{C}_{1} \subset \mathbb{R}^{2}$, and the firm, after observing its type, picks a contract in $\mathcal{C}_{1}$. (ii) The regulator observes the contract (performance) taken by the firm in the first period, and (iii) he then chooses the second-period menu $\mathcal{C}_{2}$ optimally given his beliefs about the firm's type after observing its first-period choice. (iv) Finally, the firm makes its second-period choice in $\mathcal{C}_{2}$. Let $\left(s_{1}(c(\beta)), c_{1}(\beta)\right) \in \mathcal{C}_{1}$ denote the contract taken by firm $\beta$ in the first period. Define $A(\beta)=\left\{\beta^{\prime} \in[\underline{\beta}, \bar{\beta}] \mid\left(s_{1}\left(c_{1}\left(\beta^{\prime}\right)\right), c_{1}\left(\beta^{\prime}\right)\right)=\left(s_{1}\left(c_{1}(\beta)\right), c_{1}(\beta)\right)\right\}$, the set of types that choose the same contract as type $\beta$ does in the first period. Then $\{A(\beta)\}_{\beta \in[\underline{\beta}, \bar{\beta}]}$ is a partition of the type space $[\underline{\beta}, \bar{\beta}] .{ }^{3}$ Let $\mathcal{C}_{2 A} \subset \mathbb{R}^{2}$ denote the set of contracts offered by the regulator

\footnotetext{
${ }^{3}$ Again, we assume that it is worthwhile to implement the project in either period.
} 
to segment $A$ in the second period and $\left.S_{2}\left(c_{2}(\beta \mid A)\right), c_{2}(\beta \mid A)\right) \in \mathcal{C}_{2 A}$ the contract taken by firm $\beta \in A$ in the second period. The regulator acts to maximize the discounted value of the social surplus $W_{1}+\delta W_{2}$, and the firm maximizes $\Pi_{1}+\delta \Pi_{2}$, where $\delta$ is a common discount factor with $0<\delta<1$. We use perfect Bayesian equilibrium (PBE) as our solution concept. First, we give some definitions.

Definition 1 For a given $\mathcal{C}_{1}$, a continuation equilibrium is a PBE for the subgame following $\mathcal{C}_{1}$.

Definition 2 A continuation equilibrium is called a partition equilibrium if the number of segments in the partition $\{A(\theta)\}_{\theta \in[\theta, \bar{\theta}]}$ induced by $\mathcal{C}_{1}$ is countable and each segment is connected.

Definition 3 A continuation equilibrium exhibits infinite reswitching if there exist $(s, c)$ and $\left(s^{\prime}, c^{\prime}\right)$ in $\mathcal{C}_{1}$ and an increasing sequence $\left\{\beta_{k}\right\}_{k \in \mathbb{N}}$ in $[\underline{\beta}, \bar{\beta}]$ s.t. $c_{1}\left(\beta_{2 k}\right)=c$ and $c_{1}\left(\beta_{2 k-1}\right)=c^{\prime}, k \in \mathbb{N}$.

Definition 4 For a given $\varepsilon$, a continuation equilibrium is said to exhibit pooling over a large scale $(1-\varepsilon)$ if there exists $(s, c)$ in $\mathcal{C}_{1}$ and $\underline{\beta} \leq \beta_{1} \leq \beta_{2} \leq \bar{\beta}$ s.t. $\frac{\beta_{2}-\beta_{1}}{\bar{\beta}-\underline{\beta}} \geq 1-\varepsilon$ and $c_{1}\left(\beta_{1}\right)=c_{1}\left(\beta_{2}\right)=c$

In contrast to the static model in which the optimal incentive scheme is to fully separate the types under a mild condition (see (ii) in Proposition 1), Laffont and Tirole demonstrate that in the dynamic model, for any given $\mathcal{C}_{1}$, there exists no continuation equilibrium that fully separates types in the first period. Hence some pooling is required 
in equilibrium. Moreover, they show that "much pooling" is necessary when the uncertainty is small $(\bar{\beta}-\underline{\beta}$ small), as can be seen in the following proposition. To do this, they consider a sequence of economies with fixed $\bar{\beta}$ and let the lower bound of the interval $\underline{\beta}_{n}$ converge to $\bar{\beta}$ (the density is obtained by successive truncations of the initial density).

Proposition 2 (Laffont and Tirole [3]) For any $\varepsilon>0$, there exists $\beta_{\varepsilon}<\bar{\beta}$ such that for any $n$ such that $\underline{\beta}_{n} \geq \beta_{\varepsilon}$, the equilibrium of the game on $\left[\underline{\beta}_{n}, \bar{\beta}\right]$ must either (i) involve at least a fraction $(1-\varepsilon)$ of firms producing at the same cost in the first period or (ii) exhibit both infinite reswitching and pooling over a large scale $(1-\varepsilon)$ properties.

Therefore when the uncertainty is small, this proposition tells us that the equilibrium must either be almost a fully pooling equilibrium or exhibit some sort of complexity (reswitching) and pooling over a large scale. They construct a nonpartition continuation equilibrium for a given menu with two first-period contracts $\mathcal{C}_{1}^{N P}=\{(s, c),(\widetilde{s}, \widetilde{c})\}$ which exhibits both infinite reswitching and pooling over a large scale properties and make the following conjecture [3, p.1164]:

It can also be shown that $c$ and $\widetilde{c}$ can be chosen arbitrarily close (by choos$\operatorname{ing} \widetilde{\beta}$ close to $\underline{\beta}$ ); hence, a priori, this equilibrium need not be suboptimal for small uncertainty.

In the following, however, we show that this conjecture is incorrect. First, we introduce the nonpartition continuation equilibrium that they construct. Assume the game has the following structure: a quadratic disutility of effort $\psi(e)=e^{2} / 2, \bar{\beta}-\beta=1$ and the 
prior density $f_{1}$ is uniform on $[\beta, \bar{\beta}]: f_{1}=1$. The regulator offers two contracts in the first period: $\mathcal{C}_{1}^{N P}=\{(s, c),(\widetilde{s}, \widetilde{c})\}$ with $c<\widetilde{c}$ and $s>\widetilde{s}$. These two contracts are set to extract all surplus from type $\bar{\beta}$, therefore we have the condition $s-\psi(\bar{\beta}-c)=\widetilde{s}-\psi(\bar{\beta}-\widetilde{c})=0$. In this nonpartition continuation equilibrium, there is some $\widetilde{\beta} \in(\underline{\beta}, \bar{\beta})$ such that firms in $[\underline{\beta}, \widetilde{\beta}]$ take contract $(s, c)$, and firms in $[\widetilde{\beta}, \bar{\beta}]$, who are indifferent between $(s, c)$ and $(\widetilde{s}, \widetilde{c})$ in equilibrium, take contract $(s, c)$ with probability $q=\frac{\delta \lambda}{1+\lambda} \frac{\widetilde{\beta}-\underline{\beta}}{\widetilde{c}-c} \in(0,1)$ and contract $(\widetilde{s}, \widetilde{c})$ with probability $1-q \cdot{ }^{4}$ Let $\sigma(\beta)$ denote the probability firm $\beta$ chooses $c$ in the first period. Then in this nonpartition continuation equilibrium

$$
\sigma(\beta)= \begin{cases}1 & \text { if } \beta \in[\underline{\beta}, \widetilde{\beta}] \\ q=\frac{\delta \lambda}{1+\lambda} \frac{\widetilde{\beta}-\underline{\beta}}{\widetilde{c}-c} & \text { if } \beta \in[\widetilde{\beta}, \bar{\beta}]\end{cases}
$$

It can be readily seen that this continuation equilibrium exhibits infinite reswitching and pooling over a large scale $(1-\varepsilon)$ properties.

In order to show that the nonpartition continuation equilibrium induced by $\mathcal{C}_{1}^{N P}$ is not optimal, we consider two alternative menus, $\mathcal{C}_{1}^{P_{1}}$ and $\mathcal{C}_{1}^{P_{2}}$. First let us describe $\mathcal{C}_{1}^{P_{1}}$ and its continuation equilibrium. $\mathcal{C}_{1}^{P_{1}}$ consist of two contracts: $\mathcal{C}_{1}^{P_{1}}=\left\{\left(s^{P_{1}}, c\right),\left(\widetilde{s}^{P_{1}}, \widetilde{c}\right)\right\}$, where $\widetilde{s}^{P_{1}}=\widetilde{s}=\psi(\bar{\beta}-\widetilde{c})$ and $s^{P_{1}}$ is determined by the equation $s^{P_{1}}-\frac{(\widetilde{\beta}-c)^{2}}{2}=\widetilde{s}^{P_{1}}-\frac{(\widetilde{\beta}-\widetilde{c})^{2}}{2}+$ $\delta \Pi_{2}(\widetilde{\beta} \mid \widetilde{c}) . \Pi_{2}(\widetilde{\beta} \mid \widetilde{c})$ is the profit firm $\widetilde{\beta}$ can get in the second period if he takes $\left(\widetilde{s}^{P_{1}}, \widetilde{c}\right)$ in the first period. By comparing $\mathcal{C}_{1}^{N P}$ and $\mathcal{C}_{1}^{P_{1}}$, we observe that $\mathcal{C}_{1}^{N P}$ and $\mathcal{C}_{1}^{P_{1}}$ share the same cost targets $c$ and $\widetilde{c}$. For $\mathcal{C}_{1}^{P_{1}}$, we have a partition continuation equilibrium with the following property: all firms in $[\underline{\beta}, \widetilde{\beta}]$ take $\left(s^{P_{1}}, c\right)$, and all firms in $[\widetilde{\beta}, \bar{\beta}]$ take $\left(\widetilde{s}^{P_{1}}, \widetilde{c}\right)$. Now

\footnotetext{
${ }^{4}$ Hence $\widetilde{\beta}$ can be any number between $\underline{\beta}$ and $\underline{\beta}+\frac{1+\lambda}{\delta \lambda}(\widetilde{c}-c)$.
} 
let us describe $\mathcal{C}_{1}^{P_{2}} \cdot \mathcal{C}_{1}^{P_{2}}$ contains only one of the two contracts in $\mathcal{C}_{1}^{N P}, \mathcal{C}_{1}^{P_{2}}=\{(s, c)\}$, and in a partition continuation equilibrium induced by $\mathcal{C}_{1}^{P_{2}}$ all firms take the contract in the first period; therefore it is a fully pooling equilibrium. In terms of the probability of firm $\beta \in[\widetilde{\beta}, \bar{\beta}]$ choosing $c$ in the first period, $\sigma(\beta)$ in $[\widetilde{\beta}, \bar{\beta}]$, these three continuation equilibria have the following properties: In the nonpartition continuation equilibrium induced by $\mathcal{C}_{1}^{N P}$, all firms in $[\widetilde{\beta}, \bar{\beta}]$ play an "interior" strategy $\sigma(\beta)=q \in(0,1)$. On the other hand, all firms in $[\widetilde{\beta}, \bar{\beta}]$ play a "corner" strategy in the two partition continuation equilibria induced by $\mathcal{C}_{1}^{P_{1}}$ and $\mathcal{C}_{1}^{P_{2}}: \sigma(\beta)=0$ in the partition continuation equilibrium induced by $\mathcal{C}_{1}^{P_{1}}$ and $\sigma(\beta)=1$ for the fully pooling continuation equilibrium induced by $\mathcal{C}_{1}^{P_{2}}$

By computing the welfare for these three continuation equilibria, we show that one or the other partition continuation equilibrium is necessarily better than the nonpartition continuation equilibrium induced by $\mathcal{C}_{1}^{N P}$. Let $W_{1}^{N P}+\delta W_{2}^{N P}$ denote the discounted value of the social surplus in the nonpartition continuation equilibrium, $W_{1}^{P_{1}}+\delta W_{2}^{P_{1}}$ the discounted value of the social surplus in the first partition continuation equilibrium, and $W_{1}^{P_{2}}+\delta W_{2}^{P_{2}}$ the discounted value of the social surplus in the fully pooling continuation equilibrium.

Lemma 1 If $W_{1}^{N P}+\delta W_{2}^{N P}-\left(W_{1}^{P_{2}}+\delta W_{2}^{P_{2}}\right) \geq 0$, then $W_{1}^{N P}+\delta W_{2}^{N P}-\left(W_{1}^{P_{1}}+\delta W_{2}^{P_{1}}\right)<0$

Proof. Please see the Appendix.

Given the first-period menu $\mathcal{C}_{1}^{N P}$ and the nonpartition continuation equilibrium induced by $\mathcal{C}_{1}^{N P}$, Lemma 1 says that we can construct two alternative first-period menus 
$\mathcal{C}_{1}^{P_{1}}$ and $\mathcal{C}_{1}^{P_{2}}$ leading to partition continuation equilibria and that the regulator's payoffs, the discounted value of the social surplus, can be improved upon by one of our two partition equilibria.

The intuition behind this result comes from the tradeoff between production efficiency and information rent. Offering more contracts in the first period allows the regulator to learn the firm's type better, and consequently alleviates the efficiency distortion in the second period. To do this, however, the regulator needs to pay a higher information rent to the high-type firm for revealing its type. The fully pooling continuation equilibrium does not separate types, and hence it has the highest efficiency distortion in the second period. The regulator, however, does not need to pay the information rent in the first period. Contrary to the fully pooling continuation equilibrium, the nonpartition continuation equilibrium allows the regulator to partially separate types by sorting some low types out and to alleviate the efficiency distortion in the second period. If the nonpartition continuation equilibrium dominates the fully pooling continuation equilibrium in the sense that it has a higher discounted social surplus, then the benefit from alleviating the efficiency distortion is larger than the cost of separating types in the first period. In this case, the first partition continuation equilibrium, which separates types further, should dominate the nonpartition continuation equilibrium. Furthermore, it is not hard to see from the proof in the Appendix that Lemma 1 holds for any interval $[\underline{\beta}, \bar{\beta}]$ with a uniform distribution. Hence the nonpartition continuation equilibrium is suboptimal even for small uncertainty. Undoubtedly our result relies a good deal on the 
quadratic structure of the social welfare function, but at least in this specific example we gain intuition into why a nonpartition continuation equilibrium could be dominated by a partition continuation equilibrium. The following proposition states our result formally.

Proposition 3 Whether the uncertainty is small or not, one of two partition continuation equilibria, induced by $\mathcal{C}_{1}^{P_{1}}$ or $\mathcal{C}_{1}^{P_{2}}$, yields a higher payoff for the principal than the nonpartition continuation equilibrium induced by $\mathcal{C}_{1}^{N P}$. Hence Laffont and Tirole's menu of contracts, giving rise to a nonpartition continuation equilibrium, is not optimal.

The difficulty in fully characterizing the set of equilibria arises from the complexity of the partition on the type space induced by arbitrary $C_{1}$. As mentioned earlier, if we are able to show that the optimal contract induces a partition continuation equilibrium, solving for the optimal contract is possible (see $[1,5])$. There are two possible approaches to deal with this issue. First, we can attempt to show that for any nonpartition continuation equilibrium there exists a partition continuation equilibrium that dominates this nonpartition continuation equilibrium as we did above. Alternatively, we can construct a sequence of partition continuation equilibria that approximates this nonpartition continuation equilibrium, making it reasonable for us to search for the optimal contract within the class of partition equilibria. Second, we notice that a nonpartition continuation equilibrium emerges as the result of the multiplicity of intersections of value functions with different contracts in $C_{1}$. To be precise, suppose we have a nonpartition continuation equilibrium with the partition $\{A(\beta)\}_{\beta \in[\underline{\beta}, \bar{\beta}]}$ induced by $C_{1}$, and the principal chooses second-period contracts optimally in each segment $A(\beta)$. Given this, a value 
function for some contract $(s, c)$ in $C_{1}$ is the locus between the agent's type $\beta$ and the maximum discounted sum of utilities that he can get conditional on $(s, c)$ being chosen by $\beta$ in the first period. Since it is a nonpartition continuation equilibrium, we must have two value functions associated with two contracts in $C_{1}$ with multiple intersections. In other words, we can say that the "dynamic" single crossing condition fails in nonpartition continuation equilibria. In this regard, perhaps we can eschew the difficulty of fully characterizing the set of equilibria by imposing a condition on the agent's objective function to guarantee that the dynamic single crossing condition holds. In that case, any continuation equilibrium induced by any $C_{1}$ is partitional, and hence we can search for the optimal contract within the class of partition equilibria.

\section{Conclusion}

For the specific example constructed by Laffont and Tirole [3], we show that, whether uncertainty is small or not, their nonpartition continuation equilibrium is not optimal. It is still unclear, however, whether an equilibrium is partitional in the general dynamic contracting problem without commitment, and work remains to be done to search for the optimal contract. We plan to pursue this line of research in the future.

\section{Appendix}

Proof of Lemma 1. First, we derive the discounted value of the social surplus for each continuation equilibrium. We then calculate the social surplus differences $W_{1}^{N P}+\delta W_{2}^{N P}-$ 
$\left(W_{1}^{P_{1}}+\delta W_{2}^{P_{1}}\right)$ and $W_{1}^{N P}+\delta W_{2}^{N P}-\left(W_{1}^{P_{2}}+\delta W_{2}^{P_{2}}\right)$.

(I) Derive $W_{1}^{N P}+\delta W_{2}^{N P}$. By the construction, the firm with type $\bar{\beta}$ is indifferent between $(s, c)$ and $(\widetilde{s}, \widetilde{c})$. Hence the following condition must hold:

$$
s-\psi(\bar{\beta}-c)=\widetilde{s}-\psi(\bar{\beta}-\widetilde{c})=0,
$$

which gives us

$$
\begin{aligned}
s & =\frac{(\bar{\beta}-c)^{2}}{2} \\
\widetilde{s} & =\frac{(\bar{\beta}-\widetilde{c})^{2}}{2}
\end{aligned}
$$

Now let $f$ and $\widetilde{f}$ denote the posterior densities given that $c$ and $\widetilde{c}$ have been chosen in the first period. We have:

$$
\begin{array}{r}
f= \begin{cases}\frac{1}{\widetilde{\beta}-\underline{\beta}+(\bar{\beta}-\widetilde{\beta}) g} & \text { if } \beta \in[\underline{\beta}, \widetilde{\beta}] \\
\frac{g}{\widetilde{\beta}-\underline{\beta}+(\bar{\beta}-\widetilde{\beta}) g} & \text { if } \beta \in[\widetilde{\beta}, \bar{\beta}]\end{cases} \\
\widetilde{f}= \begin{cases}0 & \text { if } \beta \in[\underline{\beta}, \widetilde{\beta}] \\
\frac{1}{\bar{\beta}-\widetilde{\beta}} & \text { if } \beta \in[\widetilde{\beta}, \bar{\beta}]\end{cases}
\end{array}
$$

Let $e^{*}(\cdot)$ and $\widetilde{e}^{*}(\cdot)$ denote the regulator's optimal incentive schemes in the second pe$\operatorname{riod}$ on $A=\left\{\beta^{\prime} \in[\underline{\beta}, \bar{\beta}] \mid\left(s_{1}\left(c_{1}\left(\beta^{\prime}\right)\right), c_{1}\left(\beta^{\prime}\right)\right)=(s, c)\right\}$ and $\widetilde{A}=\left\{\beta^{\prime} \in[\underline{\beta}, \bar{\beta}] \mid\left(s_{1}\left(c_{1}\left(\beta^{\prime}\right)\right), c_{1}\left(\beta^{\prime}\right)\right)=\right.$ $(\widetilde{s}, \widetilde{c})\}$. Applying (ii) in Proposition 1, we get

$$
\begin{aligned}
& e^{*}(\beta)= \begin{cases}1-\frac{\lambda}{1+\lambda}(\beta-\underline{\beta}) & \text { if } \beta \in[\underline{\beta}, \widetilde{\beta}] \\
1-\frac{\lambda}{1+\lambda}\left(\frac{\widetilde{\beta}-\underline{\beta}}{g}+\beta-\widetilde{\beta}\right) & \text { if } \beta \in[\widetilde{\beta}, \bar{\beta}]\end{cases} \\
& \widetilde{e}^{*}(\beta)=1-\frac{\lambda}{1+\lambda}(\beta-\widetilde{\beta}), \beta \in[\widetilde{\beta}, \bar{\beta}]
\end{aligned}
$$


With the use of (i) in Proposition 1, we can get

$$
\begin{aligned}
W_{1}^{N P}+\delta W_{2}^{N P}= & \int_{\underline{\beta}}^{\widetilde{\beta}}\left[u-(1+\lambda)(s+c)+s-\frac{(\beta-c)^{2}}{2}\right] d \beta+\int_{\widetilde{\beta}}^{\bar{\beta}}\left[u-(1+\lambda)(s+c)+s-\frac{(\beta-c)^{2}}{2}\right] g d \beta \\
& +\int_{\widetilde{\beta}}^{\bar{\beta}}\left[u-(1+\lambda)(\widetilde{s}+\widetilde{c})+\widetilde{s}-\frac{(\beta-\widetilde{c})^{2}}{2}\right](1-g) d \beta \\
& +\delta\left\{\int_{\widetilde{\beta}}\left[u-(1+\lambda)\left(\psi\left(e^{*}(\beta)\right)+\beta-e^{*}(\beta)\right)-\lambda \psi^{\prime}\left(e^{*}(\beta)\right)(\beta-\underline{\beta})\right] d \beta\right. \\
& +\int_{\widetilde{\beta}}\left[\left[u-(1+\lambda)\left(\psi\left(e^{*}(\beta)\right)+\beta-e^{*}(\beta)\right)\right] g-\lambda \psi^{\prime}\left(e^{*}(\beta)\right)(\widetilde{\beta}-\underline{\beta}+(\beta-\widetilde{\beta}) g)\right] d \beta \\
& \left.+\int_{\widetilde{\beta}}\left[\left[u-(1+\lambda)\left(\psi\left(\widetilde{e}^{*}(\beta)\right)+\beta-\widetilde{e}^{*}(\beta)\right)\right](1-g)-\lambda \psi^{\prime}\left(\widetilde{e}^{*}(\beta)\right)(\beta-\widetilde{\beta})(1-g)\right] d \beta\right\}
\end{aligned}
$$

(II) Derive $W_{1}^{P_{1}}+\delta W_{2}^{P_{1}}$. Denote $\Pi_{2}(\beta \mid \widetilde{c})$ to be the profit firm $\beta$ can get in the second period if he has chosen $\widetilde{c}$ in the first period. By construction, the following conditions must hold:

$$
\begin{aligned}
& \widetilde{s}^{P_{1}}-\psi(\bar{\beta}-\widetilde{c})=\widetilde{s}^{P_{1}}-\frac{(\bar{\beta}-\widetilde{c})^{2}}{2}=0, \\
& s^{P_{1}-\frac{(\widetilde{\beta}-c)^{2}}{2}}=\widetilde{s}^{P_{1}}-\frac{(\widetilde{\beta}-\widetilde{c})^{2}}{2}+\delta \Pi_{2}(\widetilde{\beta} \mid \widetilde{c})
\end{aligned}
$$

Now let $f^{P}$ and $\widetilde{f}^{P}$ denote the posterior densities given that $c$ and $\widetilde{c}$ have been chosen in the first period. We have:

$$
\begin{aligned}
& f^{P}= \begin{cases}\frac{1}{\widetilde{\beta}-\underline{\beta}} & \text { if } \beta \in[\underline{\beta}, \widetilde{\beta}] \\
0 & \text { if } \beta \in[\widetilde{\beta}, \bar{\beta}]\end{cases} \\
& \tilde{f}^{P}= \begin{cases}0 & \text { if } \beta \in[\underline{\beta}, \widetilde{\beta}] \\
\frac{1}{\bar{\beta}-\widetilde{\beta}} & \text { if } \beta \in[\widetilde{\beta}, \bar{\beta}]\end{cases}
\end{aligned}
$$


Let $e_{P_{1}}^{*}(\cdot)$ and $\widetilde{e}_{P_{1}}^{*}(\cdot)$ denote the regulator's optimal incentive schemes in the second period for the firm choosing $c$ and $\widetilde{c}$ repectively. Applying (ii) in Proposition 1, we get

$$
\begin{aligned}
& e_{P_{1}}^{*}(\beta)=1-\frac{\lambda}{1+\lambda}(\beta-\underline{\beta}), \beta \in[\underline{\beta}, \widetilde{\beta}] \\
& \widetilde{e}_{P_{1}}^{*}(\beta)=1-\frac{\lambda}{1+\lambda}(\beta-\widetilde{\beta}), \beta \in[\widetilde{\beta}, \bar{\beta}]
\end{aligned}
$$

With the use of (i) in Proposition 1, we can get

$$
\begin{aligned}
W_{1}^{P_{1}}+\delta W_{2}^{P_{1}}= & \int_{\underline{\beta}}^{\widetilde{\beta}}\left[u-(1+\lambda)\left(s^{P_{1}}+c\right)+s^{P_{1}}-\frac{(\beta-c)^{2}}{2}\right] d \beta+\int_{\widetilde{\beta}}^{\bar{\beta}}\left[u-(1+\lambda)\left(\widetilde{s}^{P_{1}}+\widetilde{c}\right)+\widetilde{s}^{P_{1}}-\frac{(\beta-\widetilde{c})^{2}}{2}\right] d \beta \\
& +\delta\left\{\int_{\underline{\beta}}^{\widetilde{\beta}}\left[u-(1+\lambda)\left(\psi\left(e_{P_{1}}^{*}(\beta)\right)+\beta-e_{P_{1}}^{*}(\beta)\right)-\lambda \psi^{\prime}\left(e_{P_{1}}^{*}(\beta)\right)(\beta-\underline{\beta})\right] d \beta\right. \\
& \left.+\int_{\widetilde{\beta}}^{\bar{\beta}}\left[u-(1+\lambda)\left(\psi\left(\widetilde{e}_{P_{1}}^{*}(\beta)\right)+\beta-\widetilde{e}_{P_{1}}^{*}(\beta)\right)-\lambda \psi^{\prime}\left(\widetilde{e}_{P_{1}}^{*}(\beta)\right)(\beta-\widetilde{\beta})\right] d \beta\right\}
\end{aligned}
$$

(III) Derive $W_{1}^{P_{2}}+\delta W_{2}^{P_{2}}$. By construction, $s=\frac{(\bar{\beta}-c)^{2}}{2}$. Since all types choose the same contract, the posterior density is equal to the prior density. Let $e_{P_{2}}^{*}(\cdot)$ denote the regulator's optimal incentive scheme in the second period. Applying (ii) in Proposition 1 we get

$$
e_{P_{2}}^{*}(\beta)=1-\frac{\lambda}{1+\lambda}(\beta-\underline{\beta}), \beta \in[\underline{\beta}, \bar{\beta}]
$$

With the use of (i) in Proposition 1, we can get

$$
\begin{aligned}
W_{1}^{P_{2}}+\delta W_{2}^{P_{2}}= & \int_{\underline{\beta}}^{\bar{\beta}}\left[u-(1+\lambda)(s+c)+s-\frac{(\beta-c)^{2}}{2}\right] d \beta \\
& +\delta \int_{\underline{\beta}}^{\bar{\beta}}\left[u-(1+\lambda)\left(\psi\left(e_{P_{2}}^{*}(\beta)\right)+\beta-e_{P_{2}}^{*}(\beta)\right)-\lambda \psi^{\prime}\left(e_{P_{2}}^{*}(\beta)\right)(\beta-\underline{\beta})\right] d \beta
\end{aligned}
$$

(IV) Calculate the difference $W_{1}^{N P}-W_{1}^{P_{1}}$. To make the calculation clear, we divide it into several steps: 
(i) First we notice that

$$
\begin{aligned}
s^{P_{1}}-s & =\frac{(\widetilde{\beta}-c)^{2}}{2}+\frac{(\bar{\beta}-\widetilde{c})^{2}}{2}-\frac{(\widetilde{\beta}-\widetilde{c})^{2}}{2}+\delta \Pi_{2}(\widetilde{\beta} \mid \widetilde{c})-\frac{(\bar{\beta}-c)^{2}}{2} \\
& =\frac{-2 \widetilde{\beta} c-2 \bar{\beta} \widetilde{c}+2 \widetilde{\beta} \widetilde{c}+2 \bar{\beta} c}{2}+\delta \Pi_{2}(\widetilde{\beta} \mid \widetilde{c}) \\
& =\delta \Pi_{2}(\widetilde{\beta} \mid \widetilde{c})-(\bar{\beta}-\widetilde{\beta})(\widetilde{c}-c)
\end{aligned}
$$

(ii) Applying the envelope theorem gives us $\Pi_{2}^{\prime}(\beta \mid \widetilde{c})=-\psi^{\prime}\left(\widetilde{e}_{P_{1}}^{*}(\beta)\right)=-\widetilde{e}_{P_{1}}^{*}(\beta)$.

Therefore

$\Pi_{2}(\widetilde{\beta} \mid \widetilde{c})=-\int_{\widetilde{\beta}}^{\bar{\beta}} \Pi_{2}^{\prime}(\beta \mid \widetilde{c}) d \beta=\int_{\widetilde{\beta}}^{\bar{\beta}} \widetilde{e}_{P_{1}}^{*}(\beta) d \beta=\int_{\widetilde{\beta}}^{\bar{\beta}}\left[1-\frac{\lambda}{1+\lambda}(\beta-\widetilde{\beta})\right] d \beta=\bar{\beta}-\widetilde{\beta}-\frac{\lambda}{1+\lambda} \frac{(\bar{\beta}-\widetilde{\beta})^{2}}{2}$

(iii) We also have

$$
\begin{aligned}
& \int_{\widetilde{\beta}}^{\bar{\beta}}\left[(1+\lambda)(\widetilde{s}+\widetilde{c})-\widetilde{s}+\frac{(\beta-\widetilde{c})^{2}}{2}\right] d \beta-\int_{\widetilde{\beta}}^{\bar{\beta}}\left[(1+\lambda)(s+c)-s+\frac{(\beta-c)^{2}}{2}\right] d \beta \\
= & \int_{\widetilde{\beta}}\left[(1+\lambda)(\widetilde{c}-c)+\lambda(\widetilde{s}-s)+\frac{-2 \beta(\widetilde{c}-c)+\widetilde{c}^{2}-c^{2}}{2}\right] d \beta \\
= & {[(1+\lambda)(\widetilde{c}-c)+\lambda(\widetilde{s}-s)](\bar{\beta}-\widetilde{\beta})-\frac{\bar{\beta}^{2}-\widetilde{\beta}^{2}}{2}(\widetilde{c}-c)+\frac{\widetilde{c}^{2}-c^{2}}{2}(\bar{\beta}-\widetilde{\beta}) } \\
= & {\left[(1+\lambda)(\widetilde{c}-c)+\lambda \frac{(\bar{\beta}-\widetilde{c})^{2}-(\bar{\beta}-c)^{2}}{2}\right](\bar{\beta}-\widetilde{\beta})-\frac{(\bar{\beta}-\widetilde{\beta})(\widetilde{c}-c)(\bar{\beta}+\widetilde{\beta}-\widetilde{c}-c)}{2} } \\
= & (\widetilde{c}-c)(\bar{\beta}-\widetilde{\beta})\left[1+\lambda-\frac{\lambda(2 \bar{\beta}-\widetilde{c}-c)+\bar{\beta}+\widetilde{\beta}-\widetilde{c}-c}{2}\right]
\end{aligned}
$$

(iv) Using results (i)-(iii), the first-period social surplus difference $W_{1}^{N P}-W_{1}^{P_{1}}$ can be simplified as:

$$
\begin{aligned}
& W_{1}^{N P}-W_{1}^{P_{1}} \\
= & \int_{\underline{\beta}}^{\widetilde{\beta}}\left[u-(1+\lambda)(s+c)+s-\frac{(\beta-c)^{2}}{2}\right] d \beta+\int_{\widetilde{\beta}}^{\bar{\beta}}\left[u-(1+\lambda)(s+c)+s-\frac{(\beta-c)^{2}}{2}\right] g d \beta
\end{aligned}
$$




$$
\begin{aligned}
& +\int_{\widetilde{\beta}}^{\bar{\beta}}\left[u-(1+\lambda)(\widetilde{s}+\widetilde{c})+\widetilde{s}-\frac{(\beta-\widetilde{c})^{2}}{2}\right](1-g) d \beta-\left\{\int_{\underline{\beta}}^{\widetilde{\beta}}\left[u-(1+\lambda)\left(s^{P_{1}}+c\right)+s^{P_{1}}-\frac{(\beta-c)^{2}}{2}\right] d \beta\right. \\
& \left.+\int_{\widetilde{\beta}}\left[u-(1+\lambda)\left(\widetilde{s} P^{P_{1}}+\widetilde{c}\right)+\widetilde{s}^{P_{1}}-\frac{(\beta-\widetilde{c})^{2}}{2}\right] d \beta\right\} \\
= & \int_{\tilde{\beta}}^{\widetilde{\beta}} \lambda\left(s^{P_{1}}-s\right) d \beta+\int_{\widetilde{\beta}}^{\bar{\beta}}\left[u-(1+\lambda)(s+c)+s-\frac{(\beta-c)^{2}}{2}\right] g d \beta-\int_{\widetilde{\beta}}^{\bar{\beta}}\left[u-(1+\lambda)(\widetilde{s}+\widetilde{c})+\widetilde{s}-\frac{(\beta-\widetilde{c})^{2}}{2}\right] g d \beta \\
= & \int_{\widetilde{\beta}} \lambda\left(s^{P_{1}}-s\right) d \beta+\int_{\widetilde{\beta}}\left[(1+\lambda)(\widetilde{s}+\widetilde{c})-\widetilde{s}+\frac{(\beta-\widetilde{c})^{2}}{2}\right] g d \beta-\int_{\widetilde{\beta}}\left[(1+\lambda)(s+c)-s+\frac{(\beta-c)^{2}}{2}\right] g d \beta \\
= & \lambda\left[\delta \Pi_{2}(\widetilde{\beta} \mid \widetilde{c})-(\bar{\beta}-\widetilde{\beta})(\widetilde{c}-c)\right](\widetilde{\beta}-\underline{\beta})+g(\widetilde{c}-c)(\bar{\beta}-\widetilde{\beta})\left[1+\lambda-\frac{\lambda(2 \bar{\beta}-\widetilde{c}-c)+\bar{\beta}+\widetilde{\beta}-\widetilde{c}-c]}{2}\right] \\
= & \lambda(\widetilde{\beta}-\underline{\beta})\left[\delta\left(\bar{\beta}-\widetilde{\beta}-\frac{\lambda}{1+\lambda} \frac{(\bar{\beta}-\widetilde{\beta})^{2}}{2}\right)-(\bar{\beta}-\widetilde{\beta})(\widetilde{c}-c)\right] \\
& +\frac{\delta \lambda}{1+\lambda} \frac{\widetilde{\beta}-\underline{\beta}}{\widetilde{c}-c}(\widetilde{c}-c)(\bar{\beta}-\widetilde{\beta})\left[1+\lambda-\frac{\lambda(2 \bar{\beta}-\widetilde{c}-c)+\bar{\beta}+\widetilde{\beta}-\widetilde{c}-c}{2}\right] \\
= & \lambda(\widetilde{\beta}-\underline{\beta})\left[\delta\left(\bar{\beta}-\widetilde{\beta}-\frac{\lambda}{1+\lambda} \frac{(\bar{\beta}-\widetilde{\beta})^{2}}{2}\right)-(\bar{\beta}-\widetilde{\beta})(\widetilde{c}-c)+\delta(\bar{\beta}-\widetilde{\beta})\left(1-\frac{\lambda(2 \bar{\beta}-\widetilde{c}-c)+\bar{\beta}+\widetilde{\beta}-\widetilde{c}-c}{2(1+\lambda)]}\right.\right. \\
= & \lambda(\widetilde{\beta}-\underline{\beta})(\bar{\beta}-\widetilde{\beta})\left[2 \delta-\frac{\lambda \delta}{1+\lambda} \frac{\bar{\beta}-\widetilde{\beta}}{2}-(\widetilde{c}-c)-\delta \frac{\lambda(2 \bar{\beta}-\widetilde{c}-c)+\bar{\beta}+\widetilde{\beta}-\widetilde{c}-c}{2(1+\lambda)}\right]
\end{aligned}
$$

(V) Calculate $W_{2}^{N P}-W_{2}^{P_{1}}$. First we notice that $e^{*}(\beta)=e_{P_{1}}^{*}(\beta)$ on $[\underline{\beta}, \widetilde{\beta}], \widetilde{e}^{*}(\beta)=$ $\widetilde{e}_{P_{1}}^{*}(\beta)$ on $[\widetilde{\beta}, \bar{\beta}]$ and $e^{*}(\beta)=\widetilde{e}^{*}(\beta)-d$ on $[\widetilde{\beta}, \bar{\beta}]$, where

$$
d=\frac{\lambda}{1+\lambda} \frac{\widetilde{\beta}-\underline{\beta}}{g}=\frac{\lambda}{1+\lambda}(\widetilde{\beta}-\underline{\beta}) \frac{(1+\lambda)(\widetilde{c}-c)}{\delta \lambda(\widetilde{\beta}-\underline{\beta})}=\frac{\widetilde{c}-c}{\delta} .
$$

Therefore,

$$
\begin{aligned}
W_{2}^{N P}-W_{2}^{P_{1}}= & \int_{\widetilde{\beta}}^{\bar{\beta}}\left[\left[u-(1+\lambda)\left(\psi\left(e^{*}(\beta)\right)+\beta-e^{*}(\beta)\right)\right] g-\lambda \psi^{\prime}\left(e^{*}(\beta)\right)(\widetilde{\beta}-\underline{\beta}+(\beta-\widetilde{\beta}) g)\right] d \beta \\
& -g \int_{\widetilde{\beta}}^{\bar{\beta}}\left[u-(1+\lambda)\left(\psi\left(\widetilde{e}^{*}(\beta)\right)+\beta-\widetilde{e}^{*}(\beta)\right)-\lambda \psi^{\prime}\left(\widetilde{e}^{*}(\beta)\right)(\beta-\widetilde{\beta})\right] d \beta \\
= & g\left[\int_{\widetilde{\beta}}^{\bar{\beta}}\left[(1+\lambda)\left(\psi\left(\widetilde{e}^{*}(\beta)\right)-\widetilde{e}^{*}(\beta)\right)+\lambda \psi^{\prime}\left(\widetilde{e}^{*}(\beta)\right)(\beta-\widetilde{\beta})\right] d \beta\right.
\end{aligned}
$$




$$
\left.-\int_{\widetilde{\beta}}^{\bar{\beta}}\left[(1+\lambda)\left(\psi\left(e^{*}(\beta)\right)-e^{*}(\beta)\right)+\lambda \psi^{\prime}\left(e^{*}(\beta)\right)(\beta-\widetilde{\beta})\right] d \beta\right]-\int_{\widetilde{\beta}}^{\bar{\beta}} \lambda \psi^{\prime}\left(e^{*}(\beta)\right)(\widetilde{\beta}-\underline{\beta}) d \beta
$$

Since

$$
\begin{aligned}
& \int_{\widetilde{\beta}}^{\bar{\beta}}\left[(1+\lambda)\left(\psi\left(\widetilde{e}^{*}(\beta)\right)-\widetilde{e}^{*}(\beta)\right)+\lambda \psi^{\prime}\left(\widetilde{e}^{*}(\beta)\right)(\beta-\widetilde{\beta})\right]-\left[(1+\lambda)\left(\psi\left(e^{*}(\beta)\right)-e^{*}(\beta)\right)+\lambda \psi^{\prime}\left(e^{*}(\beta)\right)(\beta-\widetilde{\beta})\right] d \beta \\
= & \int_{\widetilde{\beta}}^{\bar{\beta}}\left[(1+\lambda)\left(\frac{\left(\widetilde{e}^{*}(\beta)\right)^{2}}{2}-\widetilde{e}^{*}(\beta)\right)+\lambda \widetilde{e}^{*}(\beta)(\beta-\widetilde{\beta})\right]-\left[(1+\lambda)\left(\frac{\left(e^{*}(\beta)\right)^{2}}{2}-e^{*}(\beta)\right)+\lambda e^{*}(\beta)(\beta-\widetilde{\beta})\right] d \beta \\
= & \int_{\widetilde{\beta}}^{\bar{\beta}}\left[(1+\lambda)\left(\frac{\left(\widetilde{e}^{*}(\beta)\right)^{2}}{2}-\widetilde{e}^{*}(\beta)\right)+\lambda \widetilde{e}^{*}(\beta)(\beta-\widetilde{\beta})\right]-\left[(1+\lambda)\left(\frac{\left(\widetilde{e}^{*}(\beta)-d\right)^{2}}{2}-\widetilde{e}^{*}(\beta)+d\right)\right. \\
& \left.+\lambda\left(\widetilde{e}^{*}(\beta)-d\right)(\beta-\widetilde{\beta})\right] d \beta \\
= & -\int_{\widetilde{\beta}}^{\bar{\beta}}\left[(1+\lambda)\left(\frac{-2 \widetilde{e}^{*}(\beta) d+d^{2}}{2}+d\right)-\lambda d(\beta-\widetilde{\beta})\right] d \beta \\
= & -\int_{\widetilde{\beta}}\left[(1+\lambda)\left(\frac{\lambda}{1+\lambda}(\beta-\widetilde{\beta}) d+\frac{d^{2}}{2}\right)-\lambda d(\beta-\widetilde{\beta})\right] d \beta \\
= & -\int_{\widetilde{\beta}}(1+\lambda) \frac{d^{2}}{2} d \beta=-(1+\lambda) \frac{d^{2}}{2}(\bar{\beta}-\widetilde{\beta})
\end{aligned}
$$

and

$$
\begin{aligned}
\int_{\widetilde{\beta}}^{\bar{\beta}} \lambda \psi^{\prime}\left(e^{*}(\beta)\right)(\widetilde{\beta}-\underline{\beta}) d \beta & =\lambda(\widetilde{\beta}-\underline{\beta}) \int_{\widetilde{\beta}}^{\bar{\beta}}\left[1-\frac{\lambda}{1+\lambda}\left(\frac{\widetilde{\beta}-\underline{\beta}}{g}+\beta-\widetilde{\beta}\right)\right] d \beta \\
& =\lambda(\widetilde{\beta}-\underline{\beta})\left[\left(1-\frac{\lambda}{1+\lambda} \frac{\widetilde{\beta}-\underline{\beta}}{g}\right)(\bar{\beta}-\widetilde{\beta})-\frac{\lambda}{1+\lambda} \frac{(\bar{\beta}-\widetilde{\beta})^{2}}{2}\right] \\
& =\lambda(\widetilde{\beta}-\underline{\beta})(\bar{\beta}-\widetilde{\beta})\left[1-\frac{\lambda}{1+\lambda} \frac{\widetilde{\beta}-\underline{\beta}}{g}-\frac{\lambda}{1+\lambda} \frac{\bar{\beta}-\widetilde{\beta}}{2}\right] \\
& =\lambda(\widetilde{\beta}-\underline{\beta})(\bar{\beta}-\widetilde{\beta})\left[1-\frac{\widetilde{c}-c}{\delta}-\frac{\lambda}{1+\lambda} \frac{\bar{\beta}-\widetilde{\beta}}{2}\right],
\end{aligned}
$$

the second-period social social surplus difference $W_{2}^{N P}-W_{2}^{P_{1}}$ can be simplified as:

$$
W_{2}^{N P}-W_{2}^{P_{1}}=g\left[\int_{\widetilde{\beta}}^{\bar{\beta}}\left[(1+\lambda)\left(\psi\left(\widetilde{e}^{*}(\beta)\right)-\widetilde{e}^{*}(\beta)\right)+\lambda \psi^{\prime}\left(\widetilde{e}^{*}(\beta)\right)(\beta-\widetilde{\beta})\right] d \beta\right.
$$




$$
\begin{aligned}
& \left.-\int_{\widetilde{\beta}}^{\bar{\beta}}\left[(1+\lambda)\left(\psi\left(e^{*}(\beta)\right)-e^{*}(\beta)\right)+\lambda \psi^{\prime}\left(e^{*}(\beta)\right)(\beta-\widetilde{\beta})\right] d \beta\right]-\int_{\widetilde{\beta}}^{\bar{\beta}} \lambda \psi^{\prime}\left(e^{*}(\beta)\right)(\widetilde{\beta}-\underline{\beta}) d \beta \\
= & -g(1+\lambda) \frac{d^{2}}{2}(\bar{\beta}-\widetilde{\beta})-\lambda(\widetilde{\beta}-\underline{\beta})(\bar{\beta}-\widetilde{\beta})\left[1-\frac{\widetilde{c}-c}{\delta}-\frac{\lambda}{1+\lambda} \frac{\bar{\beta}-\widetilde{\beta}}{2}\right] \\
= & -\frac{\delta \lambda}{1+\lambda} \frac{\widetilde{\beta}-\underline{\beta}}{\widetilde{c}-c}(1+\lambda) \frac{1}{2}\left(\frac{\widetilde{c}-c}{\delta}\right)^{2}(\bar{\beta}-\widetilde{\beta})-\lambda(\widetilde{\beta}-\underline{\beta})(\bar{\beta}-\widetilde{\beta})\left[1-\frac{\widetilde{c}-c}{\delta}-\frac{\lambda}{1+\lambda} \frac{\bar{\beta}-\widetilde{\beta}}{2}\right] \\
= & -\lambda(\widetilde{\beta}-\underline{\beta})(\bar{\beta}-\widetilde{\beta})\left[1-\frac{1}{2} \frac{\widetilde{c}-c}{\delta}-\frac{\lambda}{1+\lambda} \frac{\bar{\beta}-\widetilde{\beta}}{2}\right]
\end{aligned}
$$

(VI) We now simplify $W_{1}^{N P}+\delta W_{2}^{N P}-\left(W_{1}^{P_{1}}+\delta W_{2}^{P_{1}}\right)$ using the results in (IV) and (V):

$$
\begin{aligned}
& W_{1}^{N P}+\delta W_{2}^{N P}-\left(W_{1}^{P_{1}}+\delta W_{2}^{P_{1}}\right) \\
= & \lambda(\widetilde{\beta}-\underline{\beta})(\bar{\beta}-\widetilde{\beta})\left[2 \delta-\frac{\lambda \delta}{1+\lambda} \frac{\bar{\beta}-\widetilde{\beta}}{2}-(\widetilde{c}-c)-\delta \frac{\lambda(2 \bar{\beta}-\widetilde{c}-c)+\bar{\beta}+\widetilde{\beta}-\widetilde{c}-c}{2(1+\lambda)}\right] \\
& -\delta \lambda(\widetilde{\beta}-\underline{\beta})(\bar{\beta}-\widetilde{\beta})\left[1-\frac{1}{2} \frac{\widetilde{c}-c}{\delta}-\frac{\lambda}{1+\lambda} \frac{\bar{\beta}-\widetilde{\beta}}{2}\right] \\
= & \delta \lambda(\widetilde{\beta}-\underline{\beta})(\bar{\beta}-\widetilde{\beta})\left[1-\frac{\lambda}{1+\lambda} \frac{\bar{\beta}-\widetilde{\beta}}{2}-\frac{\widetilde{c}-c}{\delta}-\frac{\lambda(2 \bar{\beta}-\widetilde{c}-c)+\bar{\beta}+\widetilde{\beta}-\widetilde{c}-c}{2(1+\lambda)}+\frac{1}{2} \frac{\widetilde{c}-c}{\delta}+\frac{\lambda}{1+\lambda} \frac{\bar{\beta}-\widetilde{\beta}}{2}\right] \\
= & \delta \lambda(\widetilde{\beta}-\underline{\beta})(\bar{\beta}-\widetilde{\beta})\left[1-\frac{1}{2} \frac{\widetilde{c}-c}{\delta}-\frac{\lambda(2 \bar{\beta}-\widetilde{c}-c)+\bar{\beta}+\widetilde{\beta}-\widetilde{c}-c}{2(1+\lambda)}\right]
\end{aligned}
$$

(VII) Simplify $W_{1}^{N P}-W_{1}^{P_{2}}$. From (I), (III) and (iii) in (IV) we can get:

$$
\begin{aligned}
W_{1}^{N P}-W_{1}^{P_{2}} & =(1-g)\left\{\int_{\widetilde{\beta}}^{\bar{\beta}}\left[u-(1+\lambda)(\widetilde{s}+\widetilde{c})+\widetilde{s}-\frac{(\beta-\widetilde{c})^{2}}{2}\right]-\left[u-(1+\lambda)(s+c)+s-\frac{(\beta-c)^{2}}{2}\right] d \beta\right\} \\
& =-(1-g)(\widetilde{c}-c)(\bar{\beta}-\widetilde{\beta})\left[1+\lambda-\frac{\lambda(2 \bar{\beta}-\widetilde{c}-c)+\bar{\beta}+\widetilde{\beta}-\widetilde{c}-c}{2}\right]
\end{aligned}
$$

(VIII) Simplify $W_{2}^{N P}-W_{2}^{P_{2}}$. From (I) and (III) we have

$$
\begin{aligned}
W_{2}^{N P}-W_{2}^{P_{2}}= & \int_{\underline{\beta}}^{\widetilde{\beta}}\left[u-(1+\lambda)\left(\psi\left(e^{*}(\beta)\right)+\beta-e^{*}(\beta)\right)-\lambda \psi^{\prime}\left(e^{*}(\beta)\right)(\beta-\underline{\beta})\right] d \beta \\
& +\int_{\widetilde{\beta}}^{\bar{\beta}}\left[\left[u-(1+\lambda)\left(\psi\left(e^{*}(\beta)\right)+\beta-e^{*}(\beta)\right)\right] g-\lambda \psi^{\prime}\left(e^{*}(\beta)\right)(\widetilde{\beta}-\underline{\beta}+(\beta-\widetilde{\beta}) g)\right] d \beta
\end{aligned}
$$




$$
\begin{aligned}
& \quad+\int_{\widetilde{\beta}}^{\bar{\beta}}\left[\left[u-(1+\lambda)\left(\psi\left(\widetilde{e}^{*}(\beta)\right)+\beta-\widetilde{e}^{*}(\beta)\right)\right](1-g)-\lambda \psi^{\prime}\left(\widetilde{e}^{*}(\beta)\right)(\beta-\widetilde{\beta})(1-g)\right] d \beta \\
& \quad-\int_{\underline{\beta}}^{\bar{\beta}}\left[u-(1+\lambda)\left(\psi\left(e_{P_{2}}^{*}(\beta)\right)+\beta-e_{P_{2}}^{*}(\beta)\right)-\lambda \psi^{\prime}\left(e_{P_{2}}^{*}(\beta)\right)(\beta-\underline{\beta})\right] d \beta \\
& =\int_{\widetilde{\beta}}^{\bar{\beta}}\left[(1+\lambda)\left(\psi\left(e_{P_{2}}^{*}(\beta)\right)-e_{P_{2}}^{*}(\beta)\right)+\lambda \psi^{\prime}\left(e_{P_{2}}^{*}(\beta)\right)(\beta-\underline{\beta})\right] d \beta \\
& \quad-\int_{\widetilde{\beta}}\left[(1+\lambda)\left(\psi\left(e^{*}(\beta)\right)-e^{*}(\beta)\right) g+\lambda \psi^{\prime}\left(e^{*}(\beta)\right)(\widetilde{\beta}-\underline{\beta}+(\beta-\widetilde{\beta}) g)\right] d \beta \\
& \quad-\int_{\widetilde{\beta}}\left[(1+\lambda)\left(\psi\left(\widetilde{e}^{*}(\beta)\right)-\widetilde{e}^{*}(\beta)\right)(1-g)+\lambda \psi^{\prime}\left(\widetilde{e}^{*}(\beta)\right)(\beta-\widetilde{\beta})(1-g)\right] d \beta
\end{aligned}
$$

First, we notice that $e_{P_{2}}^{*}(\beta)=e^{*}(\beta)+d_{1}$ on $[\widetilde{\beta}, \underline{\beta}]$, where $d_{1}=\frac{\lambda}{1+\lambda} \frac{1-g}{g}(\widetilde{\beta}-\underline{\beta})$. Then

$$
\begin{aligned}
& g \int_{\widetilde{\beta}}^{\bar{\beta}}\left[(1+\lambda)\left(\psi\left(e_{P_{2}}^{*}(\beta)\right)-e_{P_{2}}^{*}(\beta)\right)+\lambda \psi^{\prime}\left(e_{P_{2}}^{*}(\beta)\right)(\beta-\underline{\beta})\right] d \beta \\
& -\int_{\widetilde{\beta}}^{\bar{\beta}}\left[(1+\lambda)\left(\psi\left(e^{*}(\beta)\right)-e^{*}(\beta)\right) g+\lambda \psi^{\prime}\left(e^{*}(\beta)\right)(\widetilde{\beta}-\underline{\beta}+(\beta-\widetilde{\beta}) g)\right] d \beta \\
= & g \int_{\widetilde{\beta}}^{\bar{\beta}}\left[(1+\lambda)\left(\frac{\left(e^{*}(\beta)+d_{1}\right)^{2}}{2}-e^{*}(\beta)-d_{1}\right)+\lambda\left(e^{*}(\beta)+d_{1}\right)(\beta-\underline{\beta})\right] d \beta \\
= & g \int_{\widetilde{\beta}}^{\bar{\beta}}\left[(1+\lambda)\left(\frac{2 e^{*}(\beta) d_{1}+d_{1}{ }^{2}}{2}-d_{1}\right)+\lambda d_{1}(\beta-\underline{\beta})-\lambda e^{*}(\beta)\left(\frac{\tilde{\beta}-\underline{\beta}}{g}+\underline{\beta}-\underline{\beta}\right)\right] d \beta \\
= & g \int_{\widetilde{\beta}}\left[e^{*}(\beta)\left((1+\lambda) d_{1}-\lambda \frac{1-g}{g}(\widetilde{\beta}-\underline{\beta})\right)+(1+\lambda)\right] d \beta \\
= & g d_{1} \int_{\widetilde{\beta}}\left[(1+\lambda)\left(\frac{\left.e_{1}^{*}(\beta)\right)^{2}}{2}-e_{1}\right)+\lambda d_{1}(\beta-\underline{\beta})\right] d \beta
\end{aligned}
$$

On the other hand, we have $e_{P_{2}}^{*}(\beta)=\widetilde{e}^{*}(\beta)+d_{2}$ on $[\widetilde{\beta}, \underline{\beta}]$, where $d_{2}=-\frac{\lambda}{1+\lambda}(\widetilde{\beta}-\underline{\beta})=$ 
$-\frac{g}{1-g} d_{1}$. Hence,

$$
\begin{aligned}
& (1-g)\left\{\int_{\widetilde{\beta}}^{\bar{\beta}}\left[(1+\lambda)\left(\psi\left(e_{P_{2}}^{*}(\beta)\right)-e_{P_{2}}^{*}(\beta)\right)+\lambda \psi^{\prime}\left(e_{P_{2}}^{*}(\beta)\right)(\beta-\underline{\beta})\right] d \beta\right. \\
& \left.-\int_{\widetilde{\beta}}\left[(1+\lambda)\left(\psi\left(\widetilde{e}^{*}(\beta)\right)-\widetilde{e}^{*}(\beta)\right)+\lambda \psi^{\prime}\left(\widetilde{e}^{*}(\beta)\right)(\beta-\widetilde{\beta})\right] d \beta\right\} \\
= & (1-g)\left\{\int_{\widetilde{\beta}}^{\bar{\beta}}\left[(1+\lambda)\left(\frac{\left(\widetilde{e}^{*}(\beta)+d_{2}\right)^{2}}{2}-\widetilde{e}^{*}(\beta)-d_{2}\right)+\lambda\left(\widetilde{e}^{*}(\beta)+d_{2}\right)(\beta-\underline{\beta})\right] d \beta\right. \\
= & (1-g) \int_{\widetilde{\beta}}^{\bar{\beta}}\left[(1+\lambda)\left(\frac{2 \widetilde{e}^{*}(\beta) d_{2}+d_{2}^{2}}{2}-d_{2}\right)+\lambda d_{2}(\beta-\underline{\beta})+\lambda \widetilde{e}^{*}(\beta)(\widetilde{\beta}-\underline{\beta})\right] d \beta \\
= & \left.(1-g) \int_{\widetilde{\beta}}^{\bar{\beta}}\left[(1+\lambda)\left(\frac{\left(\widetilde{e}^{*}(\beta)\right)^{2}}{2}-\widetilde{e}^{*}(\beta)\right)+\lambda \widetilde{e}^{*}(\beta)(\beta-\widetilde{\beta})\right] d \beta\right\} \\
= & (1-g) d_{2} \int_{\widetilde{\beta}}^{\bar{\beta}}\left[(1+\lambda)\left(\frac{d_{2}}{2}-1\right)+\lambda(\beta-\underline{\beta})\right] d \beta
\end{aligned}
$$

Using these two results, $W_{2}^{N P}-W_{2}^{P_{2}}$ can be simplified as:

$$
\begin{aligned}
W_{2}^{N P}-W_{2}^{P_{2}}= & \int_{\widetilde{\beta}}^{\bar{\beta}}\left[(1+\lambda)\left(\psi\left(e_{P_{2}}^{*}(\beta)\right)-e_{P_{2}}^{*}(\beta)\right)+\lambda \psi^{\prime}\left(e_{P_{2}}^{*}(\beta)\right)(\beta-\underline{\beta})\right] d \beta \\
& -\int_{\widetilde{\beta}}^{\bar{\beta}}\left[(1+\lambda)\left(\psi\left(e^{*}(\beta)\right)-e^{*}(\beta)\right) g+\lambda \psi^{\prime}\left(e^{*}(\beta)\right)(\widetilde{\beta}-\underline{\beta}+(\beta-\widetilde{\beta}) g)\right] d \beta \\
& -\int_{\widetilde{\beta}}^{\bar{\beta}}\left[(1+\lambda)\left(\psi\left(\widetilde{e}^{*}(\beta)\right)-\widetilde{e}^{*}(\beta)\right)(1-g)+\lambda \psi^{\prime}\left(\widetilde{e}^{*}(\beta)\right)(\beta-\widetilde{\beta})(1-g)\right] d \beta \\
= & g d_{1} \int_{\widetilde{\beta}}^{\bar{\beta}}\left[(1+\lambda)\left(\frac{d_{1}}{2}-1\right)+\lambda(\beta-\underline{\beta})\right] d \beta+(1-g) d_{2} \int_{\widetilde{\beta}}^{\bar{\beta}}\left[(1+\lambda)\left(\frac{d_{2}}{2}-1\right)+\lambda(\beta-\underline{\beta})\right] d \beta \\
= & g d_{1}\left[\int_{\widetilde{\beta}}\left[(1+\lambda)\left(\frac{d_{1}}{2}-1\right)+\lambda(\beta-\underline{\beta})\right] d \beta-\int_{\widetilde{\beta}}\left[(1+\lambda)\left(\frac{d_{2}}{2}-1\right)+\lambda(\beta-\underline{\beta})\right] d \beta\right] \\
= & g d_{1}(1+\lambda) \frac{\bar{\beta}-\widetilde{\beta}}{2}\left(d_{1}-d_{2}\right)
\end{aligned}
$$




$$
\begin{aligned}
& =g \frac{\lambda}{1+\lambda} \frac{1-g}{g}(\widetilde{\beta}-\underline{\beta})(1+\lambda) \frac{\bar{\beta}-\widetilde{\beta}}{2} \frac{\lambda}{1+\lambda} \frac{1}{g}(\widetilde{\beta}-\underline{\beta}) \\
& =\frac{1-g}{g} \frac{\lambda^{2}}{1+\lambda}(\widetilde{\beta}-\underline{\beta})^{2} \frac{\bar{\beta}-\widetilde{\beta}}{2}
\end{aligned}
$$

(IX) Combining (VII) and (VIII), $W_{1}^{N P}+\delta W_{2}^{N P}-\left(W_{1}^{P_{2}}+\delta W_{2}^{P_{2}}\right)$ can be simplified as:

$$
\begin{aligned}
& W_{1}^{N P}+\delta W_{2}^{N P}-\left(W_{1}^{P_{2}}+\delta W_{2}^{P_{2}}\right) \\
= & -(1-g)(\widetilde{c}-c)(\bar{\beta}-\widetilde{\beta})\left[1+\lambda-\frac{\lambda(2 \bar{\beta}-\widetilde{c}-c)+\bar{\beta}+\widetilde{\beta}-\widetilde{c}-c}{2}\right]+\delta \frac{1-g}{g} \frac{\lambda^{2}}{1+\lambda}(\widetilde{\beta}-\underline{\beta})^{2} \frac{\bar{\beta}-\widetilde{\beta}}{2} \\
= & -(1-g)(\widetilde{c}-c)(\bar{\beta}-\widetilde{\beta})\left[1+\lambda-\frac{\lambda(2 \bar{\beta}-\widetilde{c}-c)+\bar{\beta}+\widetilde{\beta}-\widetilde{c}-c}{2}\right] \\
& +\delta(1-g) \frac{(1+\lambda)(\widetilde{c}-c)}{\delta \lambda(\widetilde{\beta}-\underline{\beta})} \frac{\lambda^{2}}{1+\lambda}(\widetilde{\beta}-\underline{\beta})^{2} \frac{\bar{\beta}-\widetilde{\beta}}{2} \\
= & (1-g)(\widetilde{c}-c)(\bar{\beta}-\widetilde{\beta})(1+\lambda)\left[-1+\frac{\lambda(2 \bar{\beta}-\widetilde{c}-c)+\bar{\beta}+\widetilde{\beta}-\widetilde{c}-c}{2(1+\lambda)}+\frac{\lambda(\widetilde{\beta}-\underline{\beta})}{2(1+\lambda)}\right]
\end{aligned}
$$

(X) If $W_{1}^{N P}+\delta W_{2}^{N P}-\left(W_{1}^{P_{2}}+\delta W_{2}^{P_{2}}\right) \geq 0$, then from (IX) we know the following must be true:

$$
-1+\frac{\lambda(2 \bar{\beta}-\widetilde{c}-c)+\bar{\beta}+\widetilde{\beta}-\widetilde{c}-c}{2(1+\lambda)}+\frac{\lambda(\widetilde{\beta}-\underline{\beta})}{2(1+\lambda)} \geq 0
$$

Using the result in (VI), we get

$$
\begin{aligned}
& W_{1}^{N P}+\delta W_{2}^{N P}-\left(W_{1}^{P_{1}}+\delta W_{2}^{P_{1}}\right) \\
= & \delta \lambda(\widetilde{\beta}-\underline{\beta})(\bar{\beta}-\widetilde{\beta})\left[1-\frac{1}{2} \frac{\widetilde{c}-c}{\delta}-\frac{\lambda(2 \bar{\beta}-\widetilde{c}-c)+\bar{\beta}+\widetilde{\beta}-\widetilde{c}-c}{2(1+\lambda)}\right] \\
= & \delta \lambda(\widetilde{\beta}-\underline{\beta})(\bar{\beta}-\widetilde{\beta})\left[1-\frac{1}{g} \frac{\lambda(\widetilde{\beta}-\underline{\beta})}{2(1+\lambda)}-\frac{\lambda(2 \bar{\beta}-\widetilde{c}-c)+\bar{\beta}+\widetilde{\beta}-\widetilde{c}-c}{2(1+\lambda)}\right] \\
< & \delta \lambda(\widetilde{\beta}-\underline{\beta})(\bar{\beta}-\widetilde{\beta})\left[1-\frac{\lambda(\widetilde{\beta}-\underline{\beta})}{2(1+\lambda)}-\frac{\lambda(2 \bar{\beta}-\widetilde{c}-c)+\bar{\beta}+\widetilde{\beta}-\widetilde{c}-c}{2(1+\lambda)}\right] \leq 0
\end{aligned}
$$

Q.E.D. 


\section{References}

[1] B. Caillaud, C. Mezzetti, Equilibrium reserve prices in sequential ascending auctions, J Econ. Theory 117 (2004), 78-95.

[2] J. J. Laffont, J. Tirole, Using cost observation to regulate firms," J. Political Economy 94 (1986), 614-641.

[3] J. J. Laffont, J. Tirole, The dynamics of incentive contracts, Econometrica 56 (1988), $1153-1175$.

[4] P. Milgrom, I. Segal, Envelope theorems for arbitrary choice sets, Econometrica 70 (2001), 583-601.

[5] C. J. Sun, Dynamic price discrimination and quality provision based on purchase history, Working paper (2007), Ohio State University. 